\title{
REVISIÓN DE ELECTROENCEFALOGRAFÍA PORTABLE Y SU APLICABILIDAD EN NEUROCIENCIAS
}

\author{
Carolina Serna Rojas ${ }^{1}$, Sara Rios Arismendy ${ }^{2}$, John F. Ochoa Gómez ${ }^{3}$. \\ ${ }^{1}$ Carolina Serna Rojas, Bioingeniera. Facultad de ingeniería. Grupo Neuropsicología y Conducta \\ Universidad de Antioquia, Medellín-Colombia \\ ${ }^{2}$ Sara Rios Arismendy. Estudiante de Bioingeniería. Facultad de ingeniería. Grupo Neuropsicología y Conducta \\ Universidad de Antioquia, Medellín-Colombia \\ ${ }^{3}$ John Fredy Ochoa Gómez. Ingeniero de Sistemas e Informática, MsC en Ingeniería, PhD Ingeniería Electrónica. \\ Bioingeniería, Facultad de Ingeniería. Grupo Neuropsicología y Conducta. Universidad de Antioquia, Medellín- \\ Colombia
}

\section{RESUMEN}

La electroencefalografía (EEG) es una técnica que permite registrar la actividad eléctrica del cerebro y ha sido estudiada durante los últimos cien años en diferentes ámbitos de la neurociencia. En los últimos años se ha investigado y desarrollado equipos de medición que sean portables y que permitan una buena calidad de la señal, por lo cual se realizó una revisión bibliográfica de las compañías fabricantes de algunos dispositivos de electroencefalografía portable disponibles en el mercado, se exponen sus características principales, algunos trabajos encontrados que fueron realizados con los dispositivos, comparaciones entre los mismos y una discusión acerca de las ventajas y desventajas de sus características. Finalmente se concluye que a la hora de comprar un dispositivo para electroencefalografía portable es necesario tener en cuenta el uso que se le va a dar y el costobeneficio que tiene el equipo de acuerdo con sus características.

Palabras clave: EEG móvil, EEG portable, Interfaz cerebro-computador, Señal EEG

Recibido: 07 de abril de 2021. Aceptado: 08 de junio de 2021

Received: April 07, 2021. Accepted: June 08, 2021

DOI: https://doi.org/10.33571/rpolitec.v17n34a9

\section{REVIEW OF PORTABLE ELECTROENCEPHALOGRAPHY AND ITS APPLICABILITY IN NEUROSCIENCES}

\begin{abstract}
Encephalography is a technique that allows the recording of electrical activity of the brain and has been studied during the last hundred years in different areas of neuroscience. For several years, measuring equipment that are portable and that allow a good signal quality to have been researched and developed, so a literature review of the manufacturing companies of some of portable electroencephalography devices available on the market was carried out: Its main features are exposed, as well as some of the work found that were made with those, comparisons between them and a discussion about the advantages and disadvantages of their features. It is concluded that, when a portable encephalography device is bought, it's necessary to take into consideration the use that it will be having and the cost-benefit that the device has according to its features.
\end{abstract}

Keywords: Mobile EEG, Portable EEG, Brain Computer Interface, EEG signal.

Cómo citar este artículo: C. Serna, S. Rios, J.F. Ochoa. "Revisión de electroencefalografía portable y su aplicabilidad en neurociencias" Revista Politécnica, vol.17, no.34 pp.131-152, 2021. DOI: https://doi.org/10.33571/rpolitec.v17n34a9 


\section{INTRODUCCIÓN}

El electroencefalograma (EEG), una técnica de exploración del sistema nervioso central cuyos primeros reportes en humanos fueron desarrollados en 1929 por Hans Berger [1],[2], permite registrar la actividad eléctrica proveniente de la corteza cerebral generada por procesos bioquímicos directamente relacionados con la función neuronal, de manera más específica con las neuronas piramidales que, por su organización regular a lo largo de la corteza, permiten generar campos abiertos medibles desde la superficie [3], [4].

La señal EEG se mide como una diferencia de potencial entre electrodos a lo largo del cráneo, por lo que es una tecnología que abre la posibilidad de estudiar la topografía, polaridad y variación espacio-temporal de los campos eléctricos cerebrales y su relación con el funcionamiento del sistema nervioso [2], [5]-[7].

Los estudios de la dinámica cerebral mediante EEG han sido utilizados en la búsqueda de biomarcadores tempranos en enfermedades neurodegenerativas como la enfermedad de Alzheimer y Parkinson [8]-[15], biomarcadores para medir la profundidad anestésica durante cirugía [16] y, en el 2013, la Administración de Medicamentos y Alimentos de los Estados Unidos (FDA) aprobó el uso del sistema NEBA para el diagnóstico del Déficit de Atención e Hiperactividad [17].

La relación de las señales eléctricas del cerebro con el estado mental del sujeto ha sido una de las propuestas fundamentales de la investigación neurofisiológica [18], por lo que se han venido desarrollando diferentes tecnologías que permiten realizar estudios fuera del laboratorio [19], [20] con equipos portables y de fácil preparación [21] que mejoran el desarrollo de experimentos en un ámbito natural sin que se afecte el desempeño normal de estos dispositivos dentro de actividades cotidianas [20].

Estos sistemas portables deben ser capaces de manejar eficazmente los artefactos de movimiento de forma que se pueda presentar un rendimiento similar al de la configuración típica en un laboratorio [19]. Por ello, durante la última década se han producido desarrollos significativos en la tecnología de EEG portable que superan muchas limitaciones previas y prometen hacer del registro de señales en el mundo real una práctica rutinaria [20].

Por ejemplo, el desarrollo de pequeños amplificadores alimentados por batería facilita el movimiento de un dispositivo EEG de un laboratorio al mundo exterior.

Por otra parte, los datos son grabados y guardados en un disco duro portátil para su posterior análisis, o transmitidos de forma inalámbrica en tiempo real a un dispositivo portátil para su visualización en línea [20], lo que permite el desarrollo de dispositivos orientados a interfaz cerebro-computador que favorezcan realizar mediciones sin la sobreestimación que se genera en un ambiente controlado, como lo es un laboratorio, y se puedan desarrollar dispositivos que sirvan en el ambiente exterior.

Desde el 2018 se realiza un estudio en Alemania, el estudio HOMEONE, para evaluar el seguimiento de pacientes neurológicos en entornos domésticos usando EEG portable [22], el mismo grupo encargado del estudio ha publicado resultados, en término de los hallazgos reportados por clínicos y de las características de la señal con tecnología portable, comparables con los obtenidos con el EEG convencional [23].

La tecnología de consumo actualmente disponible en el mercado incluye auriculares o cascos portátiles diseñados principalmente para aplicaciones interfaz cerebro-computador (Brain Computer Interface o $\mathrm{BCl}$ ) como, por ejemplo, Emotiv (EPOC+ e Insight), Neurosky (MindWave), etc [19], [20], [24]. También existen dispositivos más pequeños, diseñados principalmente para usar en aplicaciones de monitorización psicofisiológica y de neurofeedback, como ejemplo B-Alert-X series, mBrainTrain (SMARTING), etc. [20], [24].

Estos dispositivos portables de EEG se desarrollaron principalmente para aplicaciones específicas como juegos [25] y vigilancia de la salud y no propiamente para investigación. 
Sin embargo, la validación de ellos para aplicaciones en investigación está en curso y sus inicios son prometedores. En su mayoría han sido probados registrando potenciales relacionados a eventos (EventRelated Potencials o ERPs) y principalmente en los efectos de la onda P300 [26] al ser utilizada en aplicaciones $\mathrm{BCl}[20]$ para evaluar procesos cognitivos como la atención, entre otros.

Además, una de las preocupaciones de los investigadores es que estos dispositivos de bajo costo permitan una buena calidad y fiabilidad de la señal EEG [27].

Debido al avance en la portabilidad de los equipos de EEG, el objetivo de esta revisión es mostrar la disponibilidad que se tiene en dispositivos móviles para EEG y los desarrollos que se han encontrado en el ambiente investigativo que puedan servir en un futuro en aplicaciones clínicas, por lo cual en la sección de dispositivos móviles se presentan algunos de los dispositivos más usados actualmente; en la discusión se resumen sus características y se da una idea de los avances o investigaciones que se han realizado con ellos sobre todo en el enfoque investigativo o clínico, además de algunas apreciaciones o comparaciones entre estas.

Por último, se presentan las conclusiones obtenidas de esta revisión.

\section{MATERIALES Y MÉTODOS}

Se realizó una revisión de la literatura actual en diferentes bases de datos como Science direct, Google Scholar, Scopus, IEEE y pubmed por medio de palabras clave como: EEG portable (portable EEG), EEG móvil (mobile EEG), Interfaz cerebro computador -ICC- (Brain Computer Interface -BCl-), señal EEG (EEG signal) tanto en inglés como en español. Además, se tuvo en cuenta artículos de investigación hasta veinte años antes de la fecha en la cual se realizó la revisión (2019 - 2020).

Para la inclusión de los artículos dentro de la revisión primero se leyó el resumen de cada uno y de esta forma se separaron los artículos según los siguientes criterios de inclusión: el artículo debía contener una investigación con un equipo portable de EEG o comparaciones entre equipos portables y equipos fijos de EEG, las señales registradas con el equipo debían ser de EEG, los equipos todavía debían estar en uso o en circulación a nivel internacional.

Luego se procedió a revisar los resultados y las conclusiones de los que ya habían pasado la primera fase; en este caso, se analizó la relevancia de la información aportada por cada artículo, el tipo de estudio que se realizó y se excluyeron aquellos que no presentaban información clara sobre el equipo portable utilizado, sobre el estudio realizado o si la información que aportaba no era relevante.

Por último, se complementó la información de cada equipo con la que cada empresa fabricante del equipo aportaba en su página principal, es decir, artículos donde probaron el equipo para EEG, información de fabricación o de características del equipo, etc.

\section{RESULTADOS DISPOSITIVOS MÓVILES}

En esta revisión se establecerá un enfoque en dispositivos empleados en investigación y uso clínico. Se expondrán brevemente algunas de las empresas con sus respectivos dispositivos que a la fecha se utilizan para el estudio de señales EEG fuera de un laboratorio, estudios realizados con éstos, comparaciones entre los mismos y su presencia dentro de la clínica.

\subsection{EMOTIV Inc}


EPOC, fuera del mercado, EPOC+ e Insight (Tabla 1) son dispositivos creados por la empresa Emotiv [28]. EPOC+ es un dispositivo inalámbrico y recargable de 14 canales en configuración internacional 10-20 y 2 canales de referencia ubicados en el mastoides [29] [30]. EPOC+ (ver Fig. 1) es de diseño sencillo y flexible.

Proporciona acceso a datos EEG de alta calidad y densidad pagando una licencia del software Pure-EEG de la misma compañía [21], [28].

Insight es un auricular EEG inalámbrico de 5 canales que cuenta con electrónica que está totalmente optimizada para producir señales limpias en cualquier momento y lugar, y puede ser conectado a dispositivos móviles.

La tecnología está basada en un sistema biosensor, polimérico e hidrófilo, que elimina la necesidad de una preparación extensa y de materiales conductores como geles o solución salina [31][32].

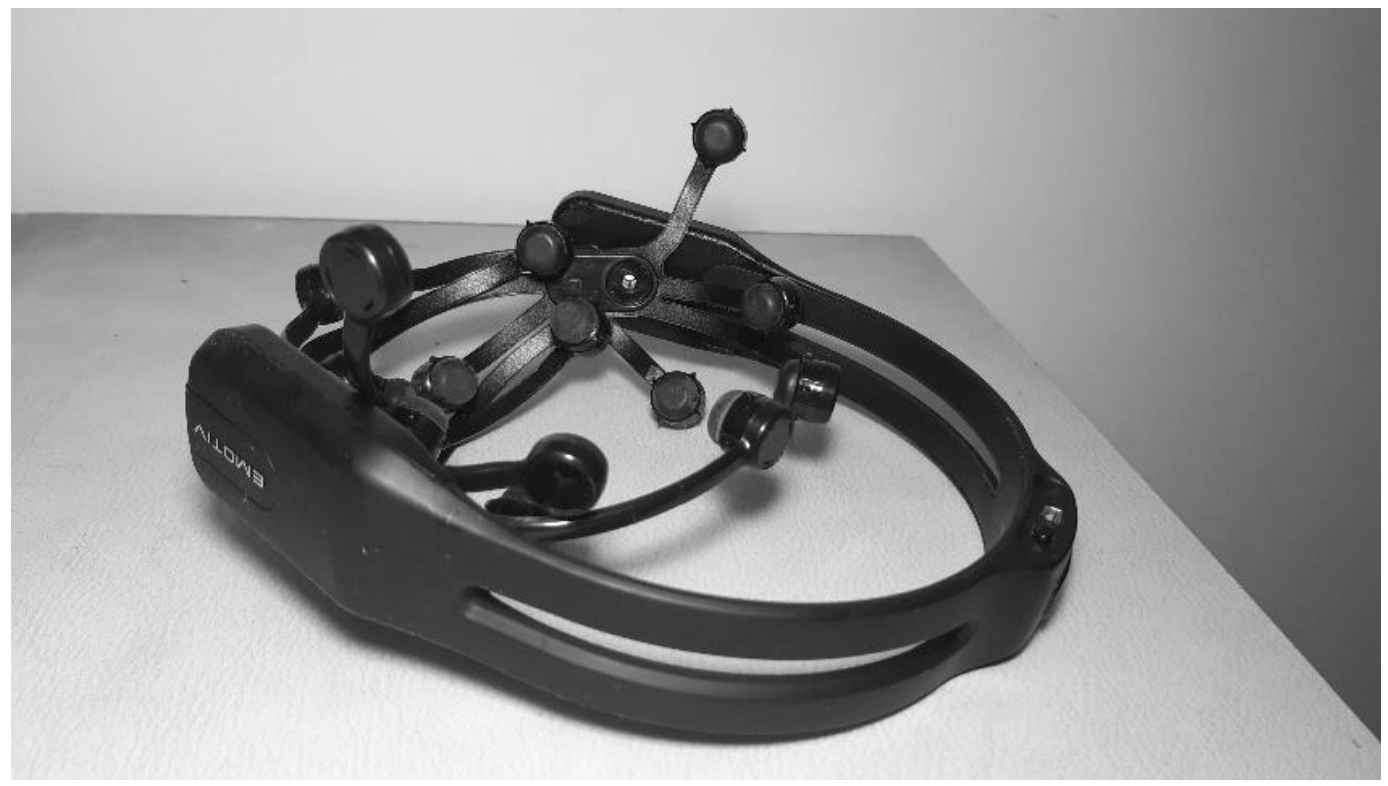

Fig. 1. Epoc+ de Emotiv.

Diferentes trabajos se han realizado con estos equipos, por ejemplo, un estudio desarrollado por Hiran Ekanayake [33] comparó el Emotiv EPOC con un equipo de 64 canales por medio de un paradigma oddball para capturar la onda P300 y lograron confirmar que el EPOC tiene una buena capacidad para captar señales EEG [33]; por otro lado, los resultados de Bahareh Nakisa et al. que compararon un dispositivo EEG de 32 canales con el equipo Emotiv Insight también confirmaron que se puede registrar señales EEG con el dispositivo móvil y a partir de éstas realizar una respectiva clasificación de emociones con características extraídas de las mismas [31].

También se ha usado en estudios más clínicos como el de Sami Schiff et al. [34] donde probaron que con un equipo de EEG portable de bajo costo se pueden obtener parámetros fiables para el diagnóstico de encefalopatía hepática [34].

Así mismo, se han desarrollado diversos estudios con la aparición de estos equipos en el mercado, como la realización de neurofeedback como alternativa de tratamiento a pacientes con Trastorno por déficit de atención con hiperactividad [21], [35]. 


\section{2. mBrainTrain}

El hardware de un EEG de 14 canales de Emotiv [28], previamente explorado por esta compañía reveló que los electrodos de éste eran una razón de la calidad moderada de la señal [36], por lo que se retiró del soporte original para conectarlo con un gorro elástico de EEG fabricado por Easycap [37] y los 14 electrodos utilizados de $\mathrm{Ag} / \mathrm{AgCl}$ fueron insertados dentro de éste para formar un sistema pequeño, con bajo peso e inalámbrico [38]. Los electrodos se ubicaron de acuerdo con la configuración 10-20 (ver Tabla 1) [39].

Esta modificación realizada dio lugar a un equipo móvil pequeño y con un amplificador ligero firmemente unido a la parte de atrás del gorro que contiene los electrodos, reduciendo en una mayor medida las interferencias presentadas por artefactos [39].

Esta tecnología fue adaptada por mBrainTrain en conjunto con EasyCap, con el deseo de convertir el primer dispositivo EEG de investigación realmente móvil, llamado SMARTING en un Smartphone. Es utilizado para una variedad de paradigmas de investigación en neurofeedback, deportes, neurorrehabilitación, neuroergonomía, entre otros y cuenta con un giroscopio en 3D [40].

Se realizó un estudio por Stefan Debener et al. [38] con este equipo, en el que se demostró que se pueden obtener datos de EEG de buena calidad en condiciones de registro adversas. Se comparó la onda P300 al estar caminando o sentado, se obtuvieron efectos confiables de la onda P300 [20], [38], presentando ondas detectables mediante una clasificación y se dio un paso hacia la grabación de un EEG en la vida diaria [39].

Por otra parte, este dispositivo fue usado en un estudio inicial para implementar un nuevo dispositivo con tecnología de EEG transparente [41]. Dicho dispositivo consiste en una matriz de sensores que permite la adquisición discreta de EEG desde los alrededores del oído.

Aunque la tecnología EEG de este tipo no se ha demostrado en la vida diaria, estudios como este se acercan cada vez más a tecnologías portables EEG que permitan hacer registros fuera de un laboratorio y con diseños cada vez más ergonómicos y cómodos [41].

\subsection{NeuroSky}

NeuroSky (neurosky.com) anunció en 2008 unos dispositivos para grabación de EEG, llamados ThinkGear, MindSet y MindBuilder [21], [30]. Sin embargo, MindSet y MindBuilder ya no se encuentran en el mercado. A la fecha esta compañía ofrece dos familias de productos EEG: para diseñadores de sistemas embebidos, se encuentra el módulo de PCG del sensor EEG de ThinkGear AM (TGAM) [21]; y para integradores de sistemas y desarrolladores de juegos, la familia MindWave que es una diadema para monitoreo de EEG [42].

La familia MindWave se compone de auriculares MindWave y MindWave Mobile [21], éste último es compatible con dispositivos móviles como iPhone, iPad y Android [43]. En el auricular MindWave Mobile (ver Tabla 1) se encuentra incluido el TGAM, que contiene además el chip TGAT, un potente sensor de EEG, y viene programado con NeuroSky eSense [44] con parámetros exclusivos para identificar procesos como atención, relajación y detección de parpadeo [45], A/D, filtro de ruido para EMG e interferencia de línea eléctrica de $50 / 60 \mathrm{~Hz}$ y permite acceder a datos crudos comprando el software [42].

Por otra parte, MindWave es un dispositivo de talla única para todos [32], donde la variabilidad en la señal y el tamaño de la cabeza cambia de acuerdo con la edad [46]. MindWave utiliza un sensor rectangular plano que debe ser presionado directamente contra la frente y emplea sensores secundarios en el lóbulo de la oreja [32], viene sin auriculares y transmite la información usando la frecuencia de radio [47].

Un enfoque utilizado en investigación con este dispositivo es la medición del parpadeo de los ojos por medio de señales de electrooculograma (EOG) [48] comúnmente visto como un artefacto de EEG. Por ello, usaron la diadema para la autenticación de parpadeos realizados por los participantes con el fin de que pueda ser fusionado con señales EEG para mejorar el desempeño de estos sistemas [48].

Además, se han realizado estudios en niños con trastorno del espectro alcohólico fetal (FASD por sus siglas en inglés) que presentan déficits cognitivos y pueden ser probados usando tareas de movimiento [49]. 


\subsection{Guger technologies (g.tec)}

Guger technologies (g.tec) es una compañía de ingeniería médica (www.gtec.at/) que proporciona soluciones para BCl. Entre los productos de g.tec se encuentran g.MOBllab+, g.Nautilus y g.Nautilus-PRO. El dispositivo g. Nautilus (ver Tabla 2) es pequeño, ligero y se une al gorro EEG para evitar los movimientos del cable favoreciendo movimientos completamente libres, permite obtener grabaciones de EEG de alta calidad desde 64 /32 /16 /8 canales en pocos minutos y tiene también la versión multipropuesta para adquirir otros tipos de bioseñales [50].

La diferencia entre éste y g. Nautilus-PRO, es que este último cuenta con certificado CE como dispositivo médico y el gorro utilizado es de 32 canales.

Por otro lado, g.MOBllab+ está disponible en dos modos: para 8 canales EEG y en el modo de adquisición multimodal, el cual puede medir otras señales fisiológicas como ECG, EMG y EOG. Se conecta mediante Bluetooth y puede durar hasta 100 horas usando cuatro baterías AA [51].

En 2016 se realizó un estudio en donde inicialmente compararon cuatro sistemas EEG (gNautilus y gMobilab fabricados por g.Tec Medical Engieneering y dos sistemas Enobio fabricados por Neuroelectrics) para identificar el hardware más confiable[52]. La mejor estabilidad de grabación fue el gNautilus por lo tanto se escogió para realizar un estudio de signos vitales del cerebro.

Para ello se seleccionaron tres ERP como indicadores iniciales: el N100 auditivo, P300 y el N400, se hicieron estos registros en adultos entre 22 a 82 años. Los resultados confirmaron ERPs específicos a nivel individual y se demostró una exitosa transformación lineal en la propuesta de signos vitales del cerebro [52].

\subsection{Advanced Brain Monitoring}

Esta compañía también ha realizado equipos inalámbricos EEG para investigación (www.advancedbrainmonitoring.com) [51], [53], como los de la serie B-Alert X-Series y Stat X-Series (ver Tabla 1). Estos dispositivos se usan para la adquisición y análisis de datos neurofisiológicos que contienen 10 o 24 electrodos dependiendo del modelo.

Los sistemas Stat X-Series son dispositivos médicos enfocados en la adquisición inalámbrica de señales EEG, EOG, ECG, y EMG, sin embargo, si el interés no es desarrollar aplicaciones EEG médicas se debe optar por el otro sistema para investigación de EEG B-Alert y el software que lo acompaña [51], [53].

Stat X-Series tiene un diseño fácil de usar con un montaje y limpieza rápida que permite realizar los procedimientos de manera eficiente y controlar la calidad de la señal en tiempo real. Los datos son guardados en formato EDF para compatibilidad universal.

El sistema Stat X-Series sólo adquiere y muestra señales fisiológicas, pero no se ha realizado ninguna demanda para el análisis de las señales adquiridas con respecto a los análisis de éstas en exactitud, precisión y fiabilidad [53].

Cada uno de los sistemas de B-Alert -X Series, entrega las mismas señales EEG y fisiológicas de alta calidad que sus homólogos Stat $X$ - Series, pero no están avalados para uso médico.

El software X-Series Basic incluye herramientas fáciles de usar para comprobar la calidad de la conexión de cada electrodo, ver datos EEG crudos en tiempo real además de insertar anotaciones con marcas de tiempo. Además, están disponibles actualizaciones opcionales de software B-Alert Integrations para aplicaciones específicas y así poder realizar mejoras de usabilidad y rendimiento [53].

Por otro lado, está la versión del B-Alert para Android que puede ser usado en una tableta y así mejorar los niveles de movilidad de las adquisiciones [51]. 
El sistema de hardware B-alert X24 se utilizó en un estudio para validar las tareas de atención y memoria que provocan ERPs para la utilización como biomarcadores en la demencia temprana [54] donde concluyeron que los ERPs obtenidos durante las tareas cognitivas pueden servir como una poderosa herramienta para evaluar el Deterioro cognitivo leve (DCL) y tener un gran potencial como biomarcadores para rastrear la progresión de la enfermedad y evaluar la respuesta terapéutica en investigación [54].

\subsection{Quasar}

Quasar (www.quasarusa.com/) ha desarrollado y lanzado una solución BCl inalámbrica. Esta solución incluye Dry Sensor Interface (DSI) 10/20 (ver Tabla 2) la adquisición de datos inalámbrica (DAQ por Data Acquisition) y un conjunto de software de su autoría. DSI 10/20 es un auricular inalámbrico BCl que está equipado con un máximo de 21 sensores EEG que son electrodos secos y proporcionan una alta impedancia de entrada adecuada para medir las señales EEG de alta fidelidad [55].

La DAQ inalámbrica es un dispositivo periférico para la transmisión de señales y grabación a bordo mediante el uso de una memoria flash. También incluye QStreamer, que es un software que contiene algoritmos de adquisición de datos, así como diferentes algoritmos de clasificación de estados cognitivos, que estiman los estados mentales del usuario en términos de carga de trabajo, compromiso y fatiga. [51], [55].

Debido a su composición electrónica de alta impedancia, no requiere preparación de la piel ni lavado de cabello luego de su utilización. Contiene un blindaje patentado y un diseño de circuitos que reducen el ruido ambiental. Se encuentra en dos versiones adicionales, una de 7 electrodos con posiciones fijas según el sistema internacional 10 - 20 y una versión de 7 electrodos con ubicación flexible. La practicidad, portabilidad y alta calidad de señal del DSI-X abre entornos de laboratorio que ahora pueden ser más naturalistas [56].

Gracias a que los sensores secos de QUASAR hacen un contacto eléctrico de alta impedancia con la piel y registran sin necesidad de preparar la piel fueron utilizados en pacientes neonatales con la idea de diagnosticar las convulsiones en dichos pacientes [57] y resultaron ser prácticos y eficientes para el uso en este tipo de pacientes.

\subsection{Starlab}

Enobio(www.neuroelectrics.com/products/enobio/) es un dispositivo inalámbrico de adquisición de EEG desarrollado por Starlab (ver Tabla 2). Este dispositivo cuenta con múltiples canales $(8,20$ y 32) y cada uno está equipado con electrodos secos. Un paquete de software (NIC) proporciona visualización en tiempo real de señales de EEG sin procesar y del espectro de potencia. Además, este sistema se ha aplicado a diversas aplicaciones asociadas con la monitorización médica, neurofeedback y el estado cognitivo [51].

Entre los posibles paradigmas $\mathrm{BCI}$ de este dispositivo se incluyen la ERD de imaginación motora, SSVEP, P300, entre otros. Enobio proporciona una plataforma que puede ser utilizada en cualquier lugar, para registrar datos de grado médico de manera rápida y confiable y realizar aplicaciones $\mathrm{BCl}$ fuera del laboratorio. La integridad de los datos, versatilidad y facilidad de uso, son las claves para el desarrollo de aplicaciones médicas y sus sistemas de recopilación de datos NUBE y NIC basados en la nube, proporcionan los medios para lograrlo de manera eficiente [58].

En el estudio realizado por E. Ratti et al. [59], se comparó la señal de EEG cuantitativamente y la fiabilidad de los sistemas de EEG de grado médico como B-Alert 24 y Enobio 20, y de consumo como Muse y MindWave. Los resultados mostraron que los datos de EEG se pueden recoger con éxito de los cuatro sistemas analizados [59].

Enobio ha sido utilizado en diversos estudios; por ejemplo, para el monitoreo del cerebro y la respuesta autónoma de niños con trastorno del espectro autista [60] en donde se pudo evidenciar cambios en la respuesta neurofisiológica y autónoma del estado de desconexión al estado de compromiso de los niños [60].

\subsection{ANT Neuro}

Es una empresa holandesa especializada en el desarrollo, marketing y ventas de aplicaciones médicas y de investigación en neurociencias y el neurodiagnóstico (www.ant-neuro.com/products/eego sports). Uno de sus 
productos es eego ${ }^{\mathrm{TM}}$ sports (ver Tabla 2), un dispositivo pensando en la movilidad que ofrece total libertad para recoger datos EEG de alta densidad, señales EMG bipolares y una variedad de datos de sensores fisiológicos en el lugar que se requiera [61].

Todos los datos grabados se almacenan directamente en la tableta que se lleva en el morral junto con el amplificador. Además, como el amplificador y la tableta están conectados directamente entre sí, el proceso de transferencia de datos no depende de la calidad de la conexión wifi o Bluetooth y, por lo tanto, los datos siempre se almacenan de forma segura en la tableta [61].

Está disponible en 4 configuraciones para grabaciones de hasta 64 canales referenciales; por otra parte, las configuraciones pro ampliadas incluyen EMG, sensores fisiológicos y adquisición de hasta 24 canales bipolares, sólo en la versión profesional. Dentro de las configuraciones escogidas por el usuario se encuentra también la ganancia programable y la frecuencia de muestreo hasta un máximo de $2048 \mathrm{~Hz}$ [62].

El dispositivo eego ${ }^{\mathrm{TM}}$ sports y el auricular Emotiv EPOC+ fueron comparados en una tarea P300 en $\mathrm{BCl}$ [63] utilizando los mismos electrodos. Los resultados sugieren que el auricular Emotiv funciona significativamente peor que el dispositivo médico (eego ${ }^{\mathrm{TM}}$ sports), además de que el auricular Emotiv tiene mayores costos relativos de operación y mantenimiento.

Este dispositivo se ha usado en algunos estudios de ambiente deportivo. En uno de ellos se utilizó para evaluar los efectos agudos de la vibración del tendón local en el flexor plantar, propiedades contráctiles musculares, actividad neuromuscular y cerebral en once jugadoras de futbol de elite de entre 15 y 16 años [64] y lograron evidenciar que la actividad cerebral inferior después de la vibración local del tendón de Aquiles, a corto plazo, puede mostrar una función cortical mejorada durante un ejercicio dinámico submáximo en mujeres jóvenes futbolistas [64].

\subsection{MITSAR}

La compañía MITSAR (www.mitsar-medical.com) también es fabricante de dispositivos para EEG, entre ellos está un EEG portable llamado Mitsar-EEG Porto, permite moverse y realizar investigaciones de EEG en cualquier lugar equipado con todos los accesorios necesarios; adicionalmente se transporta en una caja de plástico y con un SmartBCl, el cual es un EEG ambulatorio [65].

SmartBCl (ver Tabla 2) es un dispositivo inalámbrico, contiene captura de video, se puede integrar a aplicaciones $\mathrm{BCl}$, posibilita realizar actividad deportiva con él y además se utiliza en Neurofeedback y Neurorrehabilitación. Es compatible con diversos gorros de EEG como Electrocap, MCSCap, entre otros [66].

Se han realizado varios estudios con este equipo, uno de ellos tenía como objetivo evaluar el estado mental de los desarrolladores en diferentes entornos y poder así desarrollar el más adecuado para realizar diferentes tareas [67] y lograron confirmar que el nivel de atención aumenta desde un punto de vista biológico cuando se desarrolla un código en paralelo mientras que cuando se programa con música, no se observan cambios significativos en las señales.

\subsection{OpenBCl}

OpenBCl (openbci.com) se especializa en la creación de hardware de bajo costo y de alta calidad de biosensado para $\mathrm{BCl}$ (ver Tabla 1); lo que permite adaptar diferentes tipos de electrodos [68], [69]. Sus placas de biosensibilidad son compatibles con Arduino, proporcionan imágenes de alta resolución y grabación de señales EMG, ECG y EEG (ver Fig. 2) [68]. Dispone de una tarjeta programable de acuerdo con el uso que se le quiera dar (ver Fig. 2), 8 canales, ganancia programable al igual que la frecuencia de muestreo $(250 \mathrm{~Hz}$ por defecto).

El precio varía de acuerdo con la tarjeta escogida y los accesorios adquiridos [69]. 


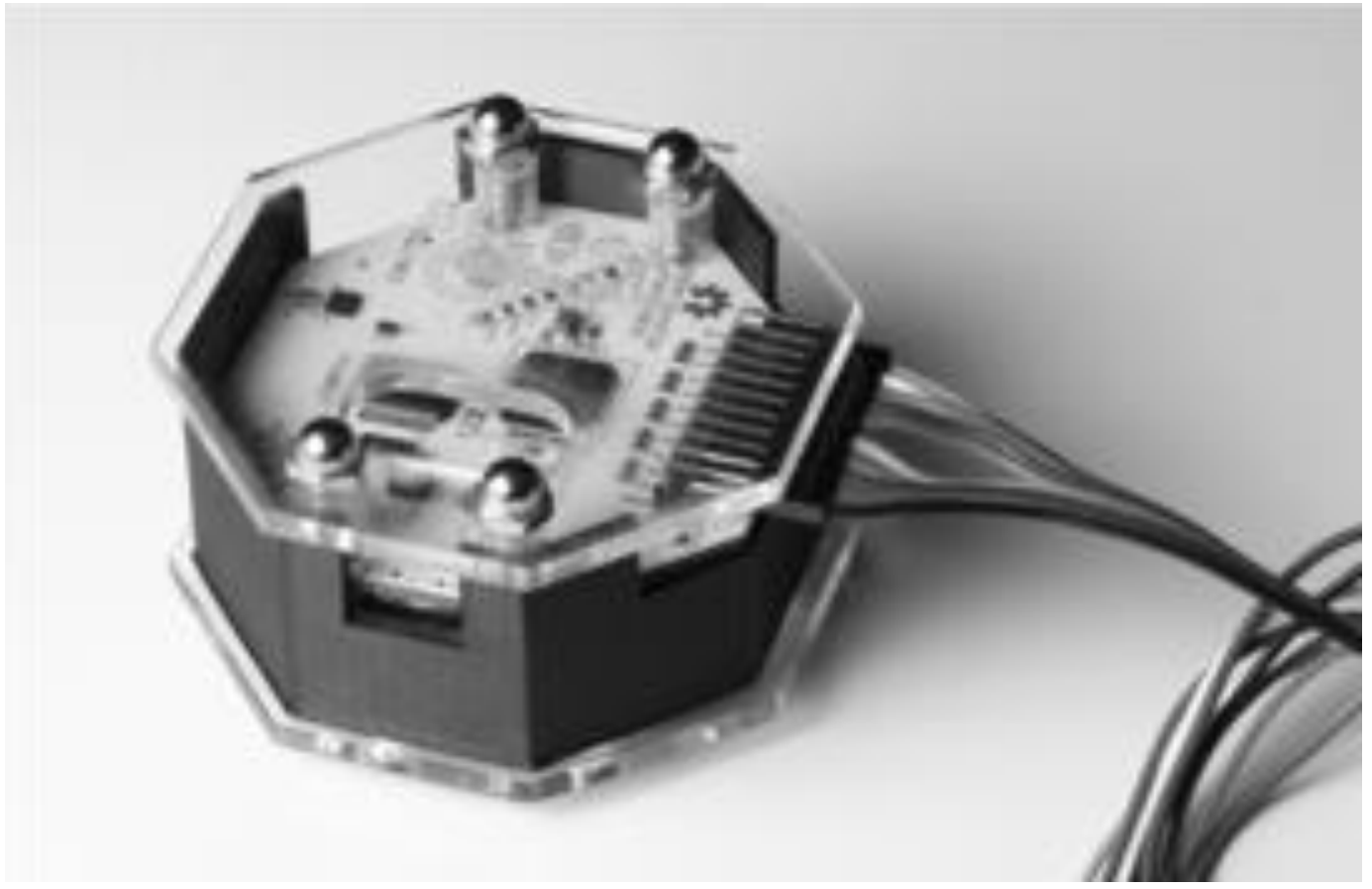

Fig. 2. Tarjeta programable OpenBCI y hardware de 8 canales.

En un estudio realizado por Jenny M. Qiu, Michael A. Casey y Solomon G. Diamond [70] utilizaron el hardware OpenBCl Cyton board con Daisy chain para evaluar científicamente la viabilidad de dicho sistema de EEG para recopilar ERP relacionados con la retroalimentación. Con este estudio llegaron a la conclusión de que el OpenBCl Cyton muestra potencial para obtener y evaluar componentes P3, N2 y FRN los cuales son indicadores de gran importancia para el monitoreo del desempeño en un entorno interactivo [70].

Diferentes estudios se pueden desarrollar con OpenBCl, como ejemplo N. Kaongoen y S. Jo [71] realizaron un estudio preliminar que introdujo la posibilidad de combinar el sistema ASSR BCl y el sistema espacial auditivo $\mathrm{BCl}$ en un sistema híbrido $\mathrm{BCl}$, para mejorar el rendimiento del $\mathrm{BCl}$ auditivo como un análogo al método $\mathrm{BCl}$ híbrido P300/SSVEP [71] y comprobaron que el sistema híbrido tuvo un mejor rendimiento que el ASSR y P300 BCl individualmente, lo que demuestra la viabilidad de incorporar ASSR y P300 espacial auditivo $\mathrm{BCl}$ en un sistema híbrido auditivo [71] y la viabilidad de utilizar este dispositivo.

También existen otros tipos de dispositivos enfocados en el entrenamiento de las personas en la relajación y la reducción del estrés, como Melomind [72] (www.mybraintech.com) y Muse de InterAxon Inc. [27], [73] (www.choosemuse.com), guiándolas a partir de sonidos cambiantes de acuerdo al estado real del cerebro por medio de EEG, basándose en el neurofeedback y los cuales se pueden conectar a un teléfono celular [68].

En la Tabla 1 y 2 se muestran las diferentes características de los dispositivos expuestos anteriormente, los cuales son todos recargables.

\section{DISCUSIÓN}

Como se ha expuesto anteriormente, existen diferentes compañías que fabrican dispositivos para investigación basados en EEG. Existen diferentes variables que los hacen diferentes y pueden generar la intriga de cuál dispositivo utilizar o cuál es la mejor opción para comprar. 
Es primordial para esto tener en cuenta qué tipo de trabajo se va a realizar, el campo en el que se va a investigar o en pocas palabras lo que se desea realizar con el dispositivo. Es recomendable tener en cuenta las características de un EEG móvil al momento de obtener el equipo (Ver Tabla 1 y 2)

Tabla 1. Comparación de los sistemas EPOC+, Insight, SMARTING, MindWave, Stat X-Series, BAlert- Series.

\begin{tabular}{|c|c|c|c|c|c|c|}
\hline $\begin{array}{c}\text { CARACTERÍS } \\
\text { TICAS }\end{array}$ & $\begin{array}{l}\text { EPOC } \\
+[28]\end{array}$ & $\begin{array}{c}\text { INSIGHT[28 } \\
]\end{array}$ & $\begin{array}{c}\text { SMARTING[ } \\
40]\end{array}$ & $\begin{array}{c}\text { MindWAV } \\
\text { E } \\
\text { MoBILE[42 } \\
]\end{array}$ & $\begin{array}{c}\text { STAT X- } \\
\text { SERIES[53] }\end{array}$ & $\begin{array}{l}\text { B-AlERT } \\
\text { SERIES[53] }\end{array}$ \\
\hline CANALES & 14 & 5 & 24 & 1 (FP1) & 9 o 20 & 9 o 20 \\
\hline $\begin{array}{l}\text { CANALES DE } \\
\text { REFERENCIA }\end{array}$ & 2 & 2 & 1 & 2 & $\begin{array}{c}1 \text { o } 4 \text { (ECG, } \\
\text { EMG, o EOG } \\
\text { ) }\end{array}$ & $\begin{array}{c}1 \text { o } 4 \text { (ECG, } \\
\text { EMG, o } \\
\text { EOG) }\end{array}$ \\
\hline $\begin{array}{c}\text { DATOS EN } \\
\text { BRUTO }\end{array}$ & $\begin{array}{c}\text { SÍ, CON } \\
\text { SUBSCRIPC } \\
\text { IÓN DE } \\
\text { SOFTWARE }\end{array}$ & $\begin{array}{c}\text { SÍ, CON } \\
\text { SUBSCRIPCI } \\
\text { ÓN DE } \\
\text { SOFTWARE } \\
\end{array}$ & Sí & $\begin{array}{c}\text { Sí, } \\
\text { COMPRAND } \\
\text { O } \\
\text { SOFTWARE }\end{array}$ & No & Sí \\
\hline ELECTRODOS & $\begin{array}{l}\text { PASTILLAS } \\
\text { DE } \\
\text { FIELTRO } \\
\text { IMPREGNA } \\
\text { DAS DE } \\
\text { SOLUCIÓN } \\
\text { SALINA } \\
\end{array}$ & $\begin{array}{l}\text { POLÍMERO } \\
\text { SEMI-SECO } \\
\text { DE LARGA } \\
\text { VIDA ÚTIL }\end{array}$ & $\begin{array}{l}\text { NECESITA LA } \\
\text { AYUDA DE } \\
\text { GEL } \\
\text { ELECTROLÍTIC } \\
\text { O }\end{array}$ & SECOS & N/A & N/A \\
\hline $\begin{array}{l}\text { RESOLUCIÓN } \\
\text { ESPACIAL }\end{array}$ & $\begin{array}{c}14 \text { o } 16 \\
\text { BITS }\end{array}$ & 14 BITS & 24 BITS & 12 BITS & 16 BITS & 16 BITS \\
\hline $\begin{array}{c}\text { SISTEMA } \\
\text { COMPATIBLE }\end{array}$ & $\begin{array}{l}\text { WINDOWS, } \\
\text { OSX, } \\
\text { LINUX, } \\
\text { ANDROID, } \\
\text { Y IOS }\end{array}$ & $\begin{array}{l}\text { WINDOWS, } \\
\text { OSX, } \\
\text { LINUX, } \\
\text { ANDROID, Y } \\
\text { IOS }\end{array}$ & $\begin{array}{l}\text { WINDOWS, } \\
\text { ANDROID }\end{array}$ & $\begin{array}{c}\text { WINDOWS } \\
\text { (XP/7/8/10 } \\
\text { ), MAC } \\
\text { (OSX } 10.8 \\
\text { O } \\
\text { POSTERIOR } \\
\text { ), IOS (IOS } \\
8 \text { O } \\
\text { POSTERIOR } \\
\text { ) AND } \\
\text { ANDROID } \\
\text { (ANDROID } \\
2.3 \text { O } \\
\text { POSTERIOR } \\
\text { ) }\end{array}$ & $\begin{array}{l}\text { WINDOWS } 8, \\
7, \text { Y XP }\end{array}$ & $\begin{array}{l}\text { WINDOWS } \\
8,7, \text { Y XP }\end{array}$ \\
\hline $\begin{array}{c}\text { FRECUENCIA } \\
\text { DE } \\
\text { RESPUESTA } \\
\end{array}$ & $\begin{array}{l}0.16- \\
43 \mathrm{~Hz}\end{array}$ & $0.5-43 \mathrm{~Hz}$ & $0-250 \mathrm{~Hz}$ & $3-100 \mathrm{~Hz}$ & $0.1-100 \mathrm{~Hz}$ & $0.1-100 \mathrm{~Hz}$ \\
\hline
\end{tabular}




\begin{tabular}{|c|c|c|c|c|c|c|}
\hline FILTRO & $\begin{array}{l}\text { DIGITAL A } \\
50 \text { Y } 60 \mathrm{~Hz}\end{array}$ & No & $\begin{array}{c}\text { ANTI- } \\
\text { ALIASING }\end{array}$ & $\begin{array}{c}\text { EMG - } \\
60 \mathrm{~Hz}\end{array}$ & N/A & N/A \\
\hline $\begin{array}{l}\text { INALÁMBRIC } \\
\text { O } \\
\text { BLUETOOTH }\end{array}$ & $\begin{array}{l}\text { SMART } \\
4.0 \mathrm{LE}\end{array}$ & $\begin{array}{l}\text { SMART } \\
\text { 4.0 LE }\end{array}$ & V2.1 & $\begin{array}{c}\text { BT/BLE } \\
\text { DUAL } \\
\text { MODE } \\
\text { MODULE } \\
\end{array}$ & $\begin{array}{l}\text { CLASS } 2 \\
+4 \mathrm{DBM} O \\
\text { ESU-MC } \\
\text { CLASS } 1 \\
\end{array}$ & $\begin{array}{l}\text { Class } 2 \\
+4 \mathrm{DBM} 0 \\
\text { ESU-MC } \\
\text { CLASS } 1\end{array}$ \\
\hline $\begin{array}{c}\text { TRANSMISIÓ } \\
\mathrm{N} \\
\text { INALÁMBRIC } \\
\mathrm{A} \\
\end{array}$ & N/A & N/A & $10 \mathrm{M}$ & $10 \mathrm{M}$ & $\sim 20 \mathrm{M}$ & $\begin{array}{c}\sim 10 \mathrm{M} \mathrm{O} \\
\sim 20 \mathrm{M}\end{array}$ \\
\hline $\begin{array}{l}\text { FRECUENCIA } \\
\text { DE MUESTREO }\end{array}$ & $\begin{array}{c}128 \text { o } 256 \\
\mathrm{~Hz}\end{array}$ & $128 \mathrm{~Hz}$ & 250 o $500 \mathrm{~Hz}$ & $512 \mathrm{~Hz}$ & $256 \mathrm{~Hz}$ & $256 \mathrm{~Hz}$ \\
\hline Costo & $\begin{array}{l}\$ 799,00+ \\
\text { ACCESORI } \\
\text { OS }\end{array}$ & $\begin{array}{c}\$ 299,00+ \\
\text { ACCESORIO } \\
\text { S }\end{array}$ & $\begin{array}{c}8,252(\mathrm{SIN} \\
\text { IVA) }\end{array}$ & $\begin{array}{c}\$ 99.99+ \\
\text { SOFTWARE } \\
(\$ 499.99)\end{array}$ & N/A & $\begin{array}{c}\sim \$ 9.950 / 19 \\
950\end{array}$ \\
\hline
\end{tabular}

Tabla 2. Comparación de los sistemas DSI 10/20, EnobioX, g.Nautilus, eego sports, SmartBCI, Avatar EEG y OpenBCI.

\begin{tabular}{|c|c|c|c|c|c|c|}
\hline $\begin{array}{c}\text { CARACTERÍSTIC } \\
\text { AS }\end{array}$ & $\begin{array}{c}\text { DSI } \\
10 / 20[5 \\
5]\end{array}$ & $\begin{array}{c}\text { ENOBIOX }[5 \\
8]\end{array}$ & $\begin{array}{c}\text { G.NAUTILUS[5 } \\
0]\end{array}$ & $\begin{array}{c}\text { EEGO }^{\text {TM }} \text { SPORTS }[ \\
61]\end{array}$ & $\begin{array}{l}\text { SMARTB } \\
\text { CI[65] }\end{array}$ & $\begin{array}{l}\text { OPENBCI[ } \\
69]\end{array}$ \\
\hline CANALES & 21 & $8 / 20 / 32$ & $8 / 16 / 32 / 64$ & $32 / 64$ & $24-32$ & 8 \\
\hline $\begin{array}{l}\text { CANALES DE } \\
\text { REFERENCIA }\end{array}$ & 3 & N/A & N/A & 6 & $\begin{array}{l}1 \text { PARA } \\
\text { ECG }\end{array}$ & N/A \\
\hline $\begin{array}{l}\text { DATOS EN } \\
\text { BRUTO }\end{array}$ & Sí & Sí & Sí & Sí & Sí & Sí \\
\hline ELECTRODOS & SECOS & SECOS & $\begin{array}{c}\text { SECOS / A BASE } \\
\text { DE GEL }\end{array}$ & A BASE DE GEL & $\begin{array}{c}\text { DEPEND } \\
\text { E DEL } \\
\text { CASCO }\end{array}$ & $\begin{array}{l}\text { DEPENDE } \\
\text { DEL CASCO }\end{array}$ \\
\hline $\begin{array}{l}\text { RESOLUCIÓN } \\
\text { ESPACIAL }\end{array}$ & 16 BITS & 24 BITS & 24 BITS & 24 BITS & 24 BITS & 24 BITS \\
\hline
\end{tabular}




\begin{tabular}{|c|c|c|c|c|c|c|}
\hline $\begin{array}{c}\text { SISTEMA } \\
\text { COMPATIBLE }\end{array}$ & $\begin{array}{l}\text { WINDO } \\
\text { WS }\end{array}$ & $\begin{array}{c}\text { WINDOWS } \\
7,8 \text { Y MAC } \\
\text { OS X }\end{array}$ & $\begin{array}{l}\text { WINDOWS Y } \\
\text { LINUX }\end{array}$ & $\begin{array}{c}8 \text { "WINDOWS 8+ } \\
\text { TABLET* }\end{array}$ & $\begin{array}{c}\text { WINDO } \\
\text { WS } \\
7 / 8 / 10 \mathrm{Y} \\
\text { OS } \\
\text { ANDRO } \\
\text { ID }\end{array}$ & $\begin{array}{l}\text { WINDOWS } \\
\text { Y MAC } \\
\text { ARDUINO/ } \\
\text { ANDROID }\end{array}$ \\
\hline $\begin{array}{l}\text { FRECUENCIA DE } \\
\text { RESPUESTA }\end{array}$ & $\begin{array}{l}0.003- \\
150 \mathrm{~Hz}\end{array}$ & $0-125 \mathrm{~Hz}$ & $0.1-40 \mathrm{~Hz}[24]$ & $\begin{array}{c}\text { DC }(0 \mathrm{~Hz})- \\
0.26^{*} \\
\text { SAMPLING } \\
\text { FREQUENCY }\end{array}$ & $\begin{array}{c}0(\mathrm{DC})- \\
70 \mathrm{~Hz}\end{array}$ & N/A \\
\hline FILTRO & $\begin{array}{c}\text { ANTI- } \\
\text { ALIASIN } \\
\text { G }\end{array}$ & N/A & N/A & N/A & N/A & N/A \\
\hline $\begin{array}{l}\text { INALÁMBRICO } \\
\text { BLUETOOTH }\end{array}$ & N/A & $\begin{array}{c}\text { BLUETOOT } \\
\text { H } 3.0 \text { AND } \\
2.1\end{array}$ & USB & USB 2 & $\begin{array}{l}\text { BLUETO } \\
\text { OTH } 2.1\end{array}$ & $\begin{array}{c}\text { BLUETOOT } \\
\text { H 4.N }\end{array}$ \\
\hline $\begin{array}{l}\text { TRANSMISIÓN } \\
\text { INALÁMBRICA }\end{array}$ & $10 \mathrm{M}$ & $\mathrm{N} / \mathrm{A}$ & $\sim 10 \mathrm{M}$ & N/A & $\sim 10 \mathrm{M}$ & N/A \\
\hline $\begin{array}{c}\text { FRECUENCIA DE } \\
\text { MUESTREO }\end{array}$ & $300 \mathrm{~Hz}$ & $500 \mathrm{~Hz}$ & $\begin{array}{c}500 \mathrm{~Hz} / \\
250 \mathrm{~Hz}(64 \\
\text { EEG) }\end{array}$ & $2048 \mathrm{~Hz}$ & $250 \mathrm{~Hz}$ & $250 \mathrm{~Hz}$ \\
\hline Costo & $\begin{array}{c}\sim \$ 22.56 \\
0\end{array}$ & N/A & $\$ \sim 5.382$ & N/A & N/A & $\begin{array}{c}\sim 199.99 \\
-949.99\end{array}$ \\
\hline
\end{tabular}

Con lo anterior se puede tener una idea a la hora de escoger un equipo que cumpla las características deseadas y permita desempeñar un EEG cómodo y confortable para el usuario.

Aunque en este texto no se exponen todos los fabricantes de dispositivos EEG móviles debido a que son una extensa cantidad y que algunos aún se encuentran en investigación, es posible prever cuáles dispositivos de los anteriormente mencionados son los más adecuados de acuerdo con las necesidades de investigación.

Un inconveniente que se presenta con frecuencia es que las investigaciones con EEG se lleva a cabo en gran medida en el laboratorio [19], pero se ha observado diferentes dispositivos como Insight, EPOC+, SMARTING y DSI 10/20 que permiten la aplicación práctica en escenarios que requieren que el sistema sea portátil y además tolere en gran medida el ruido presentado en escenarios exteriores [19] esto fomenta la investigación en neurociencias en un espacio al exterior, debido a que permiten el acceso a señales libres de algunos artefactos, facilitando el procesamiento de la señal y el desarrollo de aplicaciones $\mathrm{BCl}$. 
Sin embargo, aún se necesita investigación en el campo que permita realizar equipos para la adquisición de señales EEG en espacios abiertos con un filtrado mayor de ruido [19], [24], [75].

Emotiv y Neurosky son quizás una de las empresas con más desarrollos, no solo para aplicaciones con videojuegos, sino en investigaciones médicas como la estimación del estado de la fatiga mental [76], déficits de la memoria de trabajo en niños con trastorno del espectro alcohólico fetal [49], regulación emocional [77], imaginación motora [25], en el movimiento de una órtesis para pacientes con accidente cerebrovascular [78], detectar somnolencia o el estado de atención en conductores [79], reconocimiento de afecto o emociones [29], evaluar procesos cognitivos en personas que juegan videojuegos [80], etc.

Pero no es debido a que cuenten con certificado médico como g.Nautilus-PRO o Stat X-Series por ejemplo, sino porque son unos de los que han estado más tiempo en el mercado y han sido comparados como dispositivos para uso médico [29].

Esto genera que, en un futuro, con el desarrollo de nuevos dispositivos, se encuentren más investigaciones clínicas con el uso de equipos portátiles que tengan un certificado médico y permitan la elaboración de aplicaciones clínicas que sirvan para el diagnóstico, tratamiento o ayuda a personas que lo requieran.

Otro aspecto importante que se debe tener en cuenta a la hora de escoger el dispositivo es la cantidad de electrodos que se necesitan de acuerdo con su aplicación. Por ejemplo, los dispositivos que son en forma de diadema como los fabricados por Emotiv y NeuroSky presentan, a diferencia de los demás, menos electrodos y están principalmente ubicados en regiones occipitales (EPOC +) y oculares (MindWave), lo que sería útil en paradigmas como la P300 u otros paradigmas (o ERPs) visuales.

Sin embargo, si el objetivo no es utilizar esta zona del cerebro, se debería considerar opciones que tengan los electrodos distribuidos uniformemente a lo largo de la cabeza y que para investigación clínica podrían ser más útiles.

Este es el caso del equipo OpenBCl que permite flexibilidad con respecto a la ubicación de los electrodos, ya que pueden ser electrodos sueltos que permiten esta flexibilidad o un casco móvil. La elección de los electrodos que presenta cada uno de los equipos también es importante, porque difieren en sus características físicas y en lo que ofrecen con respecto a la relación señal-ruido.

La elección de los electrodos que presenta cada uno de los equipos también es importante, porque estos difieren en sus características físicas y en lo que ofrecen con respecto a la relación señal-ruido.

El uso de electrodos a base de gel tiene grandes ventajas, como el hecho de que estabilizan mejor la señal y al mismo tiempo reducen la impedancia de la piel; esto permite obtener mejores resultados de clasificación (mejor relación señal/ ruido) [81] a diferencia de los electrodos secos.

Por otro lado, se han realizado estudios de EEG que han demostrado que el rendimiento de estos equipos en experimentos realizados con electrodos activos es superior a los que usan electrodos pasivos, puesto que el primero presenta un pre-amplificador de la señal y evita la contaminación de esta por la suma de señales por artefactos.

Por ejemplo, para deportes más rápidos, es crucial tener un contacto muy bueno entre el cuero cabelludo y el electrodo y por lo tanto la versión de gel se recomienda para estas aplicaciones [51].

Sin embargo, existen inconvenientes con su dependencia con el contacto directo del cuero cabelludo [26]; el primero es que son inapropiados para uso a largo plazo en el registro de señales de EEG, debido a que la calidad de la señal se va degradando conforme pasa el tiempo por la regeneración de la piel y/o el secado del gel conductivo dentro de un mismo registro [24], [82] y segundo, su ubicación consume mucho tiempo, es incómoda y a menudo dolorosa para los usuarios. 
En el mercado también se pueden encontrar los electrodos secos y electrodos activos (que presentan el amplificador integrado en éste) [26] que tienen una pequeña penetración en la piel y son más recomendados [19], aunque pueden ser más costosos [82].

El problema con estos electrodos es que su capa conductora puede desaparecer al ser reutilizados varias veces, siendo más propensos al ruido y a perturbaciones, por lo cual deben ser reemplazados debido a que pueden disminuir la calidad de la señal con el tiempo [24].

Dentro de los dispositivos expuestos anteriormente, existen algunos con electrodos secos como DSI 10/20, EnobioX, g. Tec y MindWave Mobile [24].

En aplicaciones donde es menester realizar un monitoreo a largo plazo, diagnósticos médicos, actividades de la vida diaria, etc., es factible elegir un equipo discreto pero que cumpla con las condiciones necesarias donde la velocidad de aplicación sea rápida, pero sin comprometer la señal.

En cambio, si es para utilizar en un juego basado en $\mathrm{BCl}$, la discreción no es el punto fundamental, pero sí lo es su facilidad de uso y un diseño amigable al usuario [24].

También es importante tener en cuenta la disponibilidad de las señales sin procesar al momento de escoger un dispositivo de EEG portable, debido a que no todos los dispositivos presentan esta función o algunos otros como Emotiv que, aunque presenta una calidad de señal muy buena [36], necesita de una suscripción para la obtención de señales de la base de datos que supone un costo de acuerdo con el número de señales pactadas con la empresa al momento de la compra.

Para resumir, los experimentadores deben tomar decisiones informadas entre los factores mencionados para determinar qué dispositivo sería más adecuado para un estudio específico. Aunque originalmente algunos dispositivos fueron diseñados para juegos (como Emotiv y NeuroSky), una amplia variedad de aplicaciones se ha desarrollado utilizando estos equipos comerciales y que demuestran su aplicabilidad para los investigadores y la ayuda para aquellos con trastornos neuromusculares graves [32].

Por último, EEG portable es una tecnología útil para las personas con discapacidades, ya que les puede ofrecer un medio de comunicación o restablecer una función de control motor que se haya dañado. Sin embargo, es pertinente el avance de estos dispositivos que permita una mejor portabilidad, que sean más cómodos, confortables y más pequeños, de manera que se puedan adaptar a las personas fácilmente.

A pesar de la novedad de las tecnologías portables, ya se tienen reportes a nivel nacional del uso de estas, existiendo aplicaciones del OpenBCl en entornos de rehabilitación [83], anestesia [84] de derivados del NeuroSky y Emotiv [85], [86] para interfaz cerebro-computador.

En estos trabajos se extiende el papel típico del estudio de la actividad cerebral que brinda la electroencefalografía a aplicaciones novedosas, como puede ser el desarrollo de soluciones en anestesia o en el control de prótesis, indicando nuevas vías para la construcción de tecnologías médicas sustentadas por la mayor accesibilidad que permite las tecnologías portables, es por esto que podemos esperar mayores desarrollos locales en la línea del presente artículo en los próximos años.

Por último, se resalta el balance en el costo-beneficio del equipo OpenBCI, que por su bajo precio permite que sea más accesible sin perjudicar la calidad de la señal EEG.

Además, presenta una alta flexibilidad en la ubicación de electrodos, debido a que sus 8 electrodos son sueltos y facilita la ubicación en el lugar requerido, según el estudio. Además de ello, se resalta su portabilidad al ser un equipo pequeño que es fácilmente transportable.

Por otro lado, al tener una integración con arduino, su capacidad para modificar los parámetros de adquisición de la señal y la adquisición de los datos registrados permite el desarrollo de aplicaciones en neurociencias o 
en interfaz cerebro-computador que permitan el avance de la tecnología hacia equipos terapéuticos, de diagnóstico, tratamiento, etc. en el área clínica.

\section{CONCLUSIONES}

El uso de equipos portátiles para la adquisición de señales electroencefalográficas, a diferencia del EEG convencional que por sus precios no se encuentra disponible en cualquier laboratorio y por lo mismo no es sencillo desplazarse fuera de los mismos, abren la puerta a un aumento significativo de la cantidad de registros de la actividad eléctrica cerebral en diferentes entornos, algo que no solo aumentará el conocimiento sobre el funcionamiento del cerebro, sino que también abrirá nuevos campos de aplicación clínica al EEG.

En la presente revisión se han expuesto diferentes tecnologías en dispositivos portables de EEG que pueden ser usados en investigación en neurociencias y con la mayoría de las aplicaciones en interfaz cerebrocomputador.

La elección de este tipo de dispositivos depende de diferentes factores como el tipo de electrodo, el número de éstos, la comodidad del equipo, el tamaño, sus especificaciones técnicas y su relación costo-beneficio, entre otras.

En cualquier caso, como en cualquier tarea de ingeniería, es importante determinar el estudio que se va a realizar y la finalidad con la que se obtendrá el equipo y de esta manera escoger el más adecuado para sus investigaciones.

En la presente revisión se encontró que la tecnología que presenta mejor accesibilidad en precio y mayores posibilidades de adaptación es el OpenBCI por lo que se recomienda la misma para iniciar cualquier proyecto de mapeo cerebral en neurociencias usando equipos portables.

\section{AGRADECIMIENTOS}

Este trabajo se desarrolla con el apoyo de Vicerrectoría de Investigación de la Universidad de Antioquia, a través de la pasantía de Joven Investigador; así mismo, a través del proyecto CODI "Neurofisiología y Neuropsicología en Enfermedad Ganglio Basal", con código PRG2014-768.

\section{REFERENCIAS BIBLIOGRÁFICAS}

[1] B. Hans, "Uber das elektrenkephalogramm des menshen," Arch. für Psychiatr. Nervenkrankheiten, vol. 278, no. 1875, pp. 87: 527-570., 1929.

[2] F. Ramos-Argüelles, G. Morales, S. Egozcue, R. M. Pabón, and M. T. Alonso, "Técnicas básicas de electroencefalografía: principios y aplicaciones clínicas.," An. Sist. Sanit. Navar., vol. 32 Suppl 3, pp. 69-82, 2009.

[3] J. J. Vidal, "Toward Direct Brain-Computer Communication," Annual Review of Biophysics and Bioengineering, vol. 2, no. 1. pp. 157-180, 1973. 
[4] F. Lopes da Silva, "EEG and MEG: Relevance to Neuroscience," Neuron, vol. 80, no. 5, pp. 1112-1128, Dec. 2013.

[5] J. Tohka and U. Ruotsalainen, "Imaging brain change across different time scales.," Front. Neuroinform., vol. 6, p. 29, Jan. 2012.

[6] X. Zhang, X. Lei, T. Wu, and T. Jiang, "A review of EEG and MEG for brainnetome research.," Cogn. Neurodyn., vol. 8, no. 2, pp. 87-98, Apr. 2014.

[7] D. J. A. Smit et al., "Endophenotypes in a dynamically connected brain.," Behav. Genet., vol. 40, no. 2, pp. 167-77, Mar. 2010.

[8] P. M. Rossini, S. Rossi, C. Babiloni, and J. Polich, "Clinical neurophysiology of aging brain: From normal aging to neurodegeneration," Prog. Neurobiol., vol. 83, no. 6, pp. 375-400, Dec. 2007.

[9] C. Babiloni et al., "Sources of cortical rhythms change as a function of cognitive impairment in pathological aging: a multicenter study," Clin. Neurophysiol., vol. 117, no. 2, pp. 252-268, Feb. 2006.

[10] E. Gallego-Jutgla et al., "Diagnosis of Alzheimer's disease from EEG by means of synchrony measures in optimized frequency bands.," Conf. Proc. IEEE Eng. Med. Biol. Soc., vol. 2012, pp. 4266-70, Aug. 2012.

[11] C. Babiloni et al., "Classification of Single Normal and Alzheimer's Disease Individuals from Cortical Sources of Resting State EEG Rhythms," Front. Neurosci., vol. 10, p. 47, Feb. 2016.

[12] S. Nobukawa, T. Yamanishi, S. Kasakawa, H. Nishimura, M. Kikuchi, and T. Takahashi, "Classification Methods Based on Complexity and Synchronization of Electroencephalography Signals in Alzheimer's Disease," Front. Psychiatry, vol. 11, Apr. 2020.

[13] J. Carmona, J. Suarez, and J. F. Ochoa Gomez, "Brain functional connectivity in Parkinson's disease - EEG resting analysis," in IFMBE Proceedings, 2017, vol. 60, pp. 185-188.

[14] J.-M. Melgari et al., "Alpha and beta EEG power reflects L-dopa acute administration in parkinsonian patients," Front. Aging Neurosci., vol. 6, p. 302, 2014.

[15] J. F. Ochoa et al., "Precuneus Failures in Subjects of the PSEN1 E280A Family at Risk of Developing Alzheimer's Disease Detected Using Quantitative Electroencephalography.," $J$. Alzheimers. Dis., pp. 1-16, May 2017.

[16] M. Kreuzer, "EEG based monitoring of general anesthesia: Taking the next steps," Front. Comput. Neurosci., vol. 11, Jun. 2017. 
[17] A. Lenartowicz and S. K. Loo, "Use of EEG to Diagnose ADHD," Current Psychiatry Reports, vol. 16, no. 11. Current Medicine Group LLC 1, p. 498, 2014.

[18] J. J. Vidal, “Cyberspace Bionics,” 1999, pp. 203-218.

[19] J. Minguillon, M. A. Lopez-Gordo, and F. Pelayo, "Trends in EEG-BCI for daily-life: Requirements for artifact removal," Biomed. Signal Process. Control, vol. 31, pp. 407-418, 2017.

[20] J. L. Park, M. M. Fairweather, and D. I. Donaldson, "Making the case for mobile cognition: EEG and sports performance," Neurosci. Biobehav. Rev., vol. 52, no. July 2016, pp. 117-130, 2015.

[21] J. Xu and B. Zhong, "Review on portable EEG technology in educational research," Comput. Human Behav., vol. 81, pp. 340-349, 2018.

[22] T. Neumann et al., "Diagnostic and therapeutic yield of a patient-controlled portable EEG device with dry electrodes for home-monitoring neurological outpatients-rationale and protocol of the HOMEONE pilot study," Pilot Feasibility Stud., vol. 4, no. 1, Apr. 2018.

[23] T. Neumann et al., "Assessment of the technical usability and efficacy of a new portable dryelectrode EEG recorder: First results of the HOMEONE study," Clin. Neurophysiol., vol. 130, no. 11, pp. 2076-2087, Nov. 2019.

[24] V. Mihajlovic, B. Grundlehner, R. Vullers, and J. Penders, "Wearable, wireless EEG solutions in daily life applications: What are we missing?," IEEE J. Biomed. Heal. Informatics, vol. 19, no. 1, pp. 6-21, 2015.

[25] J. A. Martinez-Leon, J. M. Cano-Izquierdo, and J. Ibarrola, “Are low cost Brain Computer Interface headsets ready for motor imagery applications?," Expert Syst. Appl., vol. 49, pp. 136144, 2016.

[26] R. A. Ramadan and A. V Vasilakos, "Brain Computer Interface: Control Signals Review," Neurocomputing, vol. 223, no. October 2016, pp. 1-19, 2016.

[27] O. E. Krigolson, C. C. Williams, A. Norton, C. D. Hassall, and F. L. Colino, "Choosing MUSE: Validation of a low-cost, portable EEG system for ERP research," Front. Neurosci., vol. 11, no. MAR, pp. 1-10, 2017.

[28] EMOTIV Inc, “Homepage - Emotiv,” 2018.

[29] S. Katsigiannis and N. Ramzan, "DREAMER: A Database for Emotion Recognition Through EEG and ECG Signals from Wireless Low-cost Off-the-Shelf Devices," IEEE J. Biomed. Heal. Informatics, vol. 22, no. 1, pp. 98-107, 2018. 
[30] J. Loviscach, "Chapter 3 Playing with All Senses. Human-Computer Interface Devices for Games," Adv. Comput., vol. 77, no. 09, pp. 79-115, 2009.

[31] B. Nakisa, M. N. Rastgoo, D. Tjondronegoro, and V. Chandran, "Evolutionary computation algorithms for feature selection of EEG-based emotion recognition using mobile sensors," Expert Syst. Appl., vol. 93, pp. 143-155, 2018.

[32] J. I. Ekandem, T. A. Davis, I. Alvarez, M. T. James, and J. E. Gilbert, "Evaluating the ergonomics of BCI devices for research and experimentation," Ergonomics, vol. 55, no. 5, pp. 592-598, 2012.

[33] H. Ekanayake, "P300 and Emotiv EPOC: Does Emotiv EPOC capture real EEG?," Web Publ. http//neurofeedback. visaduma. info/ ..., p. 16, 2010.

[34] S. Schiff et al., "A low-cost, user-friendly electroencephalographic recording system for the assessment of hepatic encephalopathy," Hepatology, vol. 63, no. 5, pp. 1651-1659, 2016.

[35] Q. Wang and O. Sourina, "Real-time mental arithmetic task recognition from EEG signals," IEEE Trans. Neural Syst. Rehabil. Eng., vol. 21, no. 2, pp. 225-232, 2013.

[36] M. De Vos, M. Kroesen, R. Emkes, and S. Debener, "P300 speller BCI with a mobile EEG system: comparison to a traditional amplifier.," J. Neural Eng., vol. 11, no. 3, p. 036008, 2014.

[37] EASYCAP, “Home - EASYCAP | EEG Recording Caps and Related Products,” 2018.

[38] S. Debener, F. Minow, R. Emkes, K. Gandras, and M. de Vos, "How about taking a low-cost, small, and wireless EEG for a walk?," Psychophysiology, vol. 49, no. 11, pp. 1617-1621, 2012.

[39] M. De Vos, K. Gandras, and S. Debener, "Towards a truly mobile auditory brain-computer interface: Exploring the P300 to take away," Int. J. Psychophysiol., vol. 91, no. 1, pp. 46-53, 2014.

[40] mBrainTrain - mbt, "mBrainTrain | Smarting," 2016. .

[41] M. G. Bleichner and S. Debener, "Concealed, unobtrusive ear-centered EEG acquisition: Ceegrids for transparent EEG," Front. Hum. Neurosci., vol. 11, no. April, pp. 1-14, 2017.

[42] NeuroSky; Sitemap, “EEG - ECG - Biosensors,” 2018.

[43] NeuroSky, “A Real Game-Changer: BCI \& EEG Use Cases for Video Games,” 2015.

[44] F. Liarokapis, K. Debattista, A. Vourvopoulos, P. Petridis, and A. Ene, "Comparing interaction techniques for serious games through brain - computer interfaces: A user perception evaluation study q," Entertain. Comput., vol. 5, no. 4, pp. 391-399, 2014. 
[45] Y. Ci and S. Wang, "The key techniques research on portable EEG examination expert system," 10th Int. Conf. Comput. Sci. Educ. ICCSE 2015. Fitzwilliam Coll. Cambridge Univ. UK, no. Iccse, pp. 975-978, 2015.

[46] J. M. Rogers, S. J. Johnstone, A. Aminov, J. Donnelly, and P. H. Wilson, "Test-retest reliability of a single-channel, wireless EEG system," Int. J. Psychophysiol., vol. 106, pp. 87-96, 2016.

[47] A. Vourvopoulos and F. Liarokapis, "Evaluation of commercial brain-computer interfaces in real and virtual world environment: A pilot study," Comput. Electr. Eng., vol. 40, no. 2, pp. 714$729,2014$.

[48] M. Abo-Zahhad, S. M. Ahmed, and S. N. Abbas, "A new multi-level approach to EEG based human authentication using eye blinking," Pattern Recognit. Lett., vol. 82, pp. 216-225, 2016.

[49] K. S. Hemington and J. N. Reynolds, "Electroencephalographic correlates of working memory deficits in children with Fetal Alcohol Spectrum Disorder using a single-electrode pair recording device," Clin. Neurophysiol., vol. 125, no. 12, pp. 2364-2371, 2014.

[50] g.tec, "g.tec medical engineering," 2018.

[51] L. Seungchan, S. Younghak, W. Soogil, K. Kiseon, and L. Heung-No, "Chapter 11. Review of Wireless Brain-Computer Interface Systems," in Brain-Computer Interface Systems - Recent Progress and Future Prospects, Reza Fazel., INTECH, 2013, pp. 215-238.

[52] S. G. Hajra et al., "Developing brain vital signs: Initial framework for monitoring brain function changes over time," Front. Neurosci., vol. 10, no. MAY, pp. 1-10, 2016.

[53] Advanced Brain Monitoring Inc, “Advanced Brain Monitoring - Neurotechnology \&amp; Advanced Sleep," 2015.

[54] T. Radüntz and B. Meffert, "User experience of 7 mobile electroencephalography devices: Comparative study," JMIR mHealth uHealth, vol. 7, no. 9, 2019.

[55] Quasar, “Quasar USA,” 2016.

[56] Wearable Sensing, "Wireless Dry Electrode EEG Systems for Neuroscience Research,” 2013.

[57] I. Fridman et al., "Evaluation of Dry Sensors for Neonatal EEG Recordings," J. Clin. Neurophysiol., vol. 33, no. 2, pp. 149-155, 2016.

[58] Neuroelectrics, "Products / ENOBIO - Neuroelectrics," 2018. 
[59] E. Ratti, S. Waninger, C. Berka, G. Ruffini, and A. Verma, "Comparison of Medical and Consumer Wireless EEG Systems for Use in Clinical Trials," Front. Hum. Neurosci., vol. 11, no. August, pp. 1-7, 2017.

[60] L. Billeci et al., "An integrated approach for the monitoring of brain and autonomic response of children with Autism Spectrum Disorders during treatment by wearable technologies," Front. Neurosci., vol. 10, no. JUN, 2016.

[61] ANT Neuro, “eego ${ }^{\mathrm{TM}}$ sports $\mid$ ANT Neuro,” 2018.

[62] EGI, "Ultra-Mobile Eeg \& Emg Recording Platform,” Enschede, The Netherlands.

[63] M. Duvinage, T. Castermans, M. Petieau, T. Hoellinger, G. Cheron, and T. Dutoit, "Performance of the Emotiv Epoc headset for P300-based applications," Biomed. Eng. Online, vol. 12, no. 1, p. 56, 2013.

[64] F. Zinke, A. Gebel, U. Granacher, and O. Prieske, "Acute effects of short-term local tendon vibration on plantar flexor torque, muscle contractile properties, neuromuscular and brain activity in young athletes," J. Sport. Sci. Med., vol. 18, no. 2, pp. 327-336, 2019.

[65] Mitsar Co. Ltd, "Mitsar-EEG systems: EEG equipment for routine EEG, qEEG, ERP and video EEG studies,” 2018.

[66] MITSAR, “Electroencephalography System,” Saint Petersburg.

[67] R. Ikramov et al., "Initial evaluation of the brain activity under different software development situations," Proc. Int. Conf. Softw. Eng. Knowl. Eng. SEKE, vol. 2019-July, no. July, pp. 741-747, 2019.

[68] I. Genuth, “All In The Mind,” Eng. Technol., vol. 10, no. June, pp. 37-39, 2015.

[69] OpenBCI, “OpenBCI - Open Source Biosensing Tools (EEG, EMG, EKG, and more),” 2017.

[70] J. M. Qiu, M. A. Casey, and S. G. Diamond, “Assessing Feedback Response With a Wearable Electroencephalography System,” Front. Hum. Neurosci., vol. 13, no. July, pp. 1-14, 2019.

[71] N. Kaongoen and S. Jo, "A novel hybrid auditory BCI paradigm combining ASSR and P300," J. Neurosci. Methods, vol. 279, pp. 44-51, 2017.

[72] "myBrain Technologies," 2018.

[73] “MUSE TM | Meditation Made Easy,” 2017.

[74] Electrical Geodesics Inc, "Wireless, portable Avatar recorder for EEG, ECG, EOG, and EMG," 2018. 
[75] K. Anastasia and T. Louise, "Architecture and Neuroscience; what can the EEG recording of brain activity reveal about a walk through everyday spaces?," Int. J. Parallel, Emergent Distrib. Syst., vol. 5760, pp. 1-16, 2018.

[76] H. Xiao, Y. Duan, Z. Zhang, and M. Li, "Detection and estimation of mental fatigue in manual assembly process of complex products," Assem. Autom., p. AA-03-2017-040, 2017.

[77] A. Rodríguez, B. Rey, M. Clemente, M. Wrzesien, and M. Alcañiz, “Assessing brain activations associated with emotional regulation during virtual reality mood induction procedures," Expert Syst. Appl., vol. 42, no. 3, pp. 1699-1709, 2015.

[78] S. Fok et al., "An EEG-Based Brain Computer Interface for Rehabilitation and Restoration of Hand Control Following Stroke Using Ipsilateral Cortical Physiology," in Proceedings of the 33 annual international conference of the IEEE on engineering in medicine and biology society, EMBC,Boston, USA, 2011, pp. 6277-6280.

[79] M. Ben Dkhil, M. Neji, A. Wali, and A. M. Alimi, "A new approach for a safe car assistance system," in 20154th IEEE International Conference on Advanced logistics and Transport (ICAl T), 2015, pp. 217-222.

[80] T. Mcmahan, I. Parberry, and T. D. Parsons, "Modality Specific Assessment of Video Game Player's Experience Using the Emotiv,” Entertain. Comput., vol. 7, pp. 1-6, 2015.

[81] C. P. Amaral, M. A. Simões, S.

Mouga, J. Andrade, and M. Castelo-branco, "A novel Brain Computer Interface for classification of social joint attention in autism and comparison of 3 experimental setups: A feasibility study," vol. 290, pp. 105-115, 2017.

[82] L.-D. Liao et al., "Gaming control using a wearable and wireless EEG-based brain-computer interface device with novel dry foam-based sensors," J. Neuroeng. Rehabil., vol. 9, no. 1, p. 5, 2012.

[83] O. R. Daniela, H. I. Verónica and O. G. John, "SSVEP Study in Monocular and Binocular Vision," 2019 XXII Symposium on Image, Signal Processing and Artificial Vision (STSIVA), 2019, pp. 1-5, doi: 10.1109/STSIVA.2019.8730241.

[84] V. Gaviria García et al., "Assessment of changes in the electrical activity of the brain during general anesthesia using portable electroencephalography", Colombian Journal of Anesthesiology, vol. 49, no. 2, 2020. Available: 10.5554/22562087.e956

[85] J. Salgado Patrón and C. Barrera Monje, "Emotiv EPOC BCI with Python on a Raspberry pi", Sistemas y Telemática, vol. 14, no. 36, pp. 27-38, 2016. Available: 10.18046/syt.v14i36.2217 
[86] K. Correa Arana and O. Vivas Albán, "Prótesis de Mano Virtual Movida Por Señales Encefalograficas - EEG.", Prospectiva, vol. 14, no. 2, p. 99, 2016. Available: 10.15665/rp.v14i2.664 